\title{
Collateral ligament injuries of the metacarpophalangeal joint of the thumb: a treatment algorithm
}

\author{
Shelain Patel • Anish Potty • Emma J. Taylor • \\ Elliot D. Sorene
}

Received: 24 October 2009/Accepted: 15 January 2010/Published online: 6 February 2010

(C) Springer-Verlag 2010

\begin{abstract}
The management of injury to the ulnar and radial collateral ligaments at the metacarpophalangeal joint of the thumb is complex. Treatment is dependent upon a number of factors with a wide variety of options for each ligament. Inadequate treatment has the potential to lead to a poor functional outcome. We present the relevant clinical anatomy, mechanism of injury, methods of treatment available and suggest a single treatment algorithm for use in the management of these injuries.
\end{abstract}

Keywords Ulnar collateral ligament .

Radial collateral ligament · Thumb .

Metacarpophalangeal Joint · Algorithm

\section{Introduction}

Traumatic injuries to the hand represent a large proportion of work presenting to emergency departments and hand surgery units. Injuries to thumbs in particular deserve special attention since they perform fine, dextrous tasks as well as maintain grip. There are two main supporting ligaments traversing the metacarpophalangeal (MCP) joint of the thumb: the ulnar and radial collateral ligaments. Injuries to these ligaments can lead to symptomatic joint instability with subsequent pain, weakness and arthritis if ignored. By enlarge, injury to the ulnar collateral ligament (UCL) is more common than the radial collateral ligament (RCL) accounting for $60-90 \%$ of ligamentous collateral

S. Patel $(\bowtie) \cdot$ A. Potty $\cdot$ E. J. Taylor · E. D. Sorene

Department of Trauma and Orthopaedics,

University College Hospital, 235 Euston Road,

London NW1 2BU, UK

e-mail: shelain.patel@doctors.org.uk injury cases at the MCP joint of the thumb [1]. This review will outline the relevant clinical anatomy, mechanisms of injury, and treatment options available. This will be used to construct a treatment algorithm for managing injury to these ligaments.

\section{Anatomy and biomechanics}

The thumb MCP joint permits movement predominantly in the plane of flexion and extension; rotation, abduction and adduction are limited when compared to the other digits. The combination of the bony architecture and surrounding soft tissue support confers this increased constraint and stability. Bony stability arises from the wider, flatter metacarpal head as compared to the other digits, and also through the buttress effect of sesamoid bones that are found within the tendinous insertions of the flexor pollicis brevis and the adductor pollicis on either side of the joint [2].

The soft tissues can be thought to offer both dynamic and static stability. Dynamic stability is provided by the muscles of the thumb whilst the ulnar and radial collateral ligaments supply static stability. Both collateral ligaments are typically $4-8 \mathrm{~mm}$ in width, 12-14 $\mathrm{mm}$ in length [3-5], and give lateral support and dorsal stability for the proximal phalanx [6]. The ulnar and radial collateral ligaments arise from the medial and lateral tubercles of the metacarpal condyles respectively, and insert into the base of the proximal phalanx on their respective sides. Both ligaments consist of a proper ligament, and accessory ligament which inserts into the volar plate. Stability in MCP joint flexion is provided by the proper collateral ligaments which become taut with concomitant laxity of the accessory ligaments. Full extension of the joint confers the opposite tensioning 


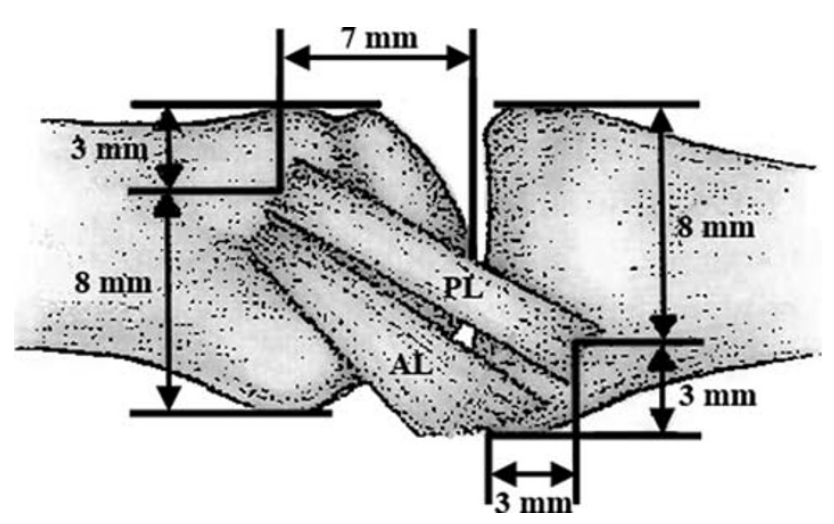

Fig. 1 The mean locations of the UCL complex which is comprised of the proper ligament (PL) and accessory ligament (AL) (adapted with permission Bean et al. 1999)

of ligaments with stability mainly provided by the accessory ligaments (see Fig. 1) [7].

It is important to be aware of the differences in the adductor and abductor aponeuroses that sit on either side of the MCP joint. The adductor aponeurosis lies in the volar position on the ulnar side of the joint, whilst the abductor aponeurosis lies in dorsal position on the radial side. Rupture of either collateral ligament will result in the ligament retracting towards its attachment and dorsally, and explains the why a Stener lesion occurs on the ulnar side only as a general rule.

A Stener lesion was first described by Bertil Stener in 1962 [8]. It refers to interposition of the adductor aponeurosis between the ruptured end of UCL and the site of its attachment on the proximal phalanx. Such interposition prevents healing at its insertion and can thus give rise to chronic instability. Stener's case series described this lesion in $64 \%$ of patients who had a complete rupture of the UCL.

\section{Mechanism of injury}

Acute injuries to the UCL occur following sudden, forced abduction movements at the MCP joint while forced adduction will injure the RCL. Repeated abduction or adduction strain will cause a chronic laxity of the respective ligament.

Acute injuries of the UCL have been termed 'Skier's thumb' after reports in skiers [9] whose thumbs were forced in to abduction and hyperextension against a ski pole or the ground. Chronic laxity injuries of the UCL meanwhile were first described by Campbell [10] amongst British gamekeepers. He noted that the act of repeatedly breaking rabbits' necks on the ground against the thumb and index finger led to UCL instability; this led to the term
'Gamekeeper's thumb'. Within the literature, these two terms have been interchangeable between acute and chronic injuries though they should ideally be separated and only used in their appropriate context.

The classic presentation of RCL injuries meanwhile is during sporting activity where the thumb is struck by a ball or player, or after a fall on to the radial aspect of the thumb and hand [11].

\section{Clinical presentation}

A presenting complaint of pain and swelling at the MCP joint following an appropriate history of trauma or repeated strain should alert the clinician to the possibility of a collateral ligament injury. Patients with chronic injuries frequently mention a history of weakness of pincer grip, holding a pen, grasping objects, unscrewing jar lids, and turning a key or door knob [12, 13].

Clinical examination may demonstrate bruising, swelling and tenderness at the MCP joint, whilst a torn ligament that has retracted back to its bony attachment is sometimes felt as a distinct mass. A targeted examination is then required to ascertain the severity of the injury such that management can be directed towards either a non-operative or operative route.

Firstly, the metacarpal is stabilised with one hand to provide a support against which the proximal phalanx can pivot. The MCP joint is then flexed to isolate the proper collateral ligament and a varus or valgus strain applied to identify injury to the radial or ulnar collateral ligament, respectively. The amount of flexion required for examination and degree of angulation that indicates a complete tear of the proper collateral ligament varies between authors (see Table 1). Since measuring angulation without a goniometer, and comparing the expected angulation to the contralateral thumb are both unreliable [14], we recommend that stress examination demonstrating a firm end-point indicates an incomplete tear [15] whilst opening of the MCP joint without a firm end point indicates a complete tear. The MCP joint is then fully extended and the same procedure performed with angulation up to the same limits as described now indicative of combined tears of the proper and accessory ligaments. With UCL injuries, testing in extension with findings compatible of a complete tear may be associated with a Stener lesion in over $85 \%$ of cases [16]. A Stener lesion itself might also be palpated as a ligamentous lump sitting outside of the adductor aponeurosis [17].

Pain when examining can cause apprehension with subsequent tensing of the surrounding muscles; this can lead to a false negative result. In such cases, local anaesthetic can be infiltrated around the site of injury to improve diagnostic accuracy [18]. 
Table 1 A table demonstrating wide variability in different authors' definition of clinical evidence of a complete ligamentous tear

\begin{tabular}{llll}
\hline Study & Collateral ligament & Position of thumb & Diagnosis of injury \\
\hline Frank and Dobyns [5] & UCL and RCL & Not stated & $\begin{array}{c}\text { For UCL: }>45^{\circ} \\
\text { For RCL: }>30^{\circ} \text { for men, }>25^{\circ} \\
\text { for women and children }\end{array}$ \\
Smith [25] & & Full flexion and extension & $>45^{\circ}$ than the normal side \\
Bowers [52] & UCL and RCL & $25^{\circ}$ flexion and extension & $>20^{\circ}$ absolute or $>10^{\circ}$ than \\
the normal side & $>35^{\circ}$ absolute \\
Palmer and Louis [53] & UCL & Full flexion & $>35^{\circ}$ absolute \\
Louis et al. [22] & UCL & Full flexion & $>45^{\circ}$ than the normal side \\
Derkash et al. [54] & UCL & Not stated & $>30^{\circ}$ absolute \\
Hintermann et al. [23] & UCL & Full flexion & $>30^{\circ}$ than the normal side \\
Musharafieh et al. [55] & UCL & $30^{\circ}$ flexion and extension & UCL
\end{tabular}

\section{Investigations}

Following history and examination, standard PA and lateral radiographs of the thumb should be requested to identify bony avulsion injuries, rule out other fractures and look for associated radiological signs of injury. This includes the PA radiograph showing deviation of the thumb away from the injured side due to the pull of the contralateral collateral ligament (see Figs. 2, 3). Volar subluxation may be visualised on the lateral radiograph for two reasons. Firstly a functionally deficient collateral ligament can allow the adductor pollicis (which is found on the volar surface) to act as a deforming force in this direction, and secondly because of associated dorsal capsular tears (see Fig. 4).

When a diagnosis is certain, no further investigations are needed and treatment can be initiated. Where the diagnosis

Fig. 2 PA radiograph of the thumb showing marked radial deviation compatible with a complete tear of the UCL

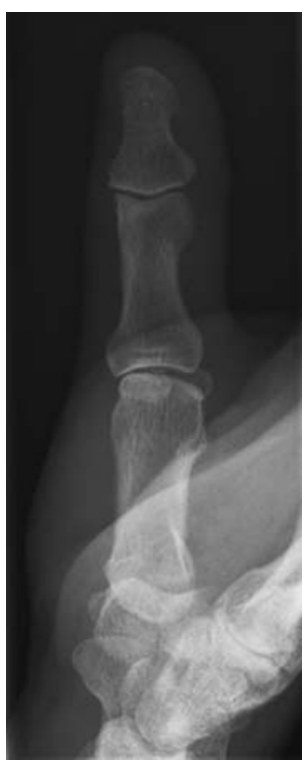

Fig. 3 PA radiograph of the thumb showing mild ulnar deviation compatible with an incomplete tear of the RCL

is uncertain, stress radiographs have historically been ordered after plain radiographs with angulation of the proximal phalanx suggestive of injury. However, stressing the joint has the potential to propagate any underlying injury and convert a partial tear into a complete tear. Accordingly, when stress radiography is not used and diagnosis is limited by a patient's pain, ultrasound [19] and MRI [20] are effective imaging tools for a proper diagnosis especially in cases of unclear clinical situations. They are especially useful for identifying Stener lesions and differentiating between partial and complete which would have implications for treatment. They are both non-invasive and readily available and thus also provide a valuable adjunct medico legally for residents in training who may have little experience of this injury. 
Fig. 4 Lateral radiograph of the thumb showing volar subluxation compatible with a complete tear of a collateral ligament at the MCP joint

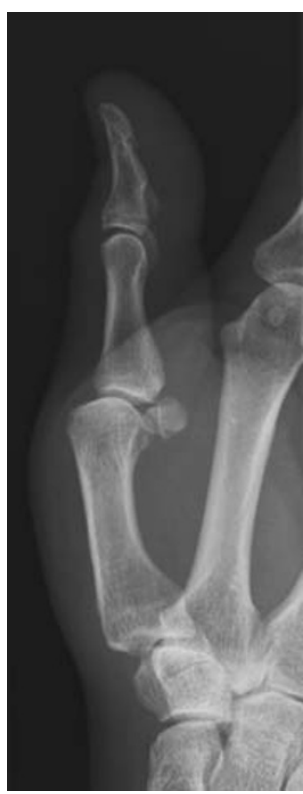

Table 3 Pechlaner's classification of UCL injuries [24]

\begin{tabular}{ll}
\hline Type & Injury \\
\hline 0 & $\begin{array}{c}\text { Individual fibres are ruptured causing elongation of the } \\
\text { ligament but compromise to the continuity }\end{array}$ \\
I & $\begin{array}{c}\text { Complete intrasubstance ligament rupture leaving proximal } \\
\text { and distal stumps }\end{array}$ \\
II $\quad \begin{array}{c}\text { Avulsion of the ligament from either the proximal or distal } \\
\text { attachments }\end{array}$ & $\begin{array}{c}\text { Bony avulsion of the ligament from either the proximal or } \\
\text { distal attachments }\end{array}$ \\
III
\end{tabular}

Pechlaner [24] meanwhile classified the injury into four types though this classification is rarely used (see Table 3 ).

\section{Management}

Once the nature of the injury has been delineated, a decision is needed as to whether non-operative or operative treatment should be employed. Smith [25] advocated that acute incomplete tears-now termed Grade I or II-are managed non-operatively with surgery indicated:

- When a Stener lesion is suspected

- When a displaced avulsion fracture exists

- In an acute, grossly unstable joint

- In a symptomatic chronic injury

- In cases of volar subluxation seen on radiographs

Hintermann [21] suggested that for his classification of UCL injuries:

- Type I injuries require cast immobilisation for 4 weeks.

- Types III and V injuries require cast immobilisation for 3 weeks.

- Types II and IV injuries require operative management.

There are instances where the injury does not fall between these two groups and then clinical judgement is needed to decide the best course of action.

\section{Non-operative treatment}

The mainstay of non-operative treatment is firstly immobilisation of the joint followed by targeted hand therapy. The duration of this immobilisation is a balance between providing enough time to allow the ligaments or an undisplaced fracture to heal and not being immobilised for so long that a joint stiffness ensues.

Most authors suggest that immobilisation is required for 3-6 weeks though up to 12 weeks has been described [26]. It is not known whether such a prolonged period is 
associated with better or worse short and long-term outcomes and no studies have compared outcome with immobilisation time.

The choice of immobilisation device is dependent upon local health resources. Specialised functional splints exist that allow flexion and extension of the MCP joint but prevent ulnar and radial deviation of the thumb. They have been shown to be more comfortable for patients when compared against the traditional plaster cast whilst not affecting stability, range of motion, strength, or duration of sick leave [27]. They are however, more expensive than plaster casts. Our institution immobilises patients for 4 weeks in a plaster cast followed by 2 weeks in a thermoplastic splint. We feel this offers the best compromise between initial joint immobilisation and early, limited range of motion to prevent long-term stiffness.

\section{Operative treatment}

Operative approach and primary repair

Exposure is similar for both the UCL and RCL and the authors' approach is now outlined. An incision measuring 4-5 cm centred over the MCP joint is made. For access to the UCL, the incision is curved toward the dorsoulnar aspect whilst for access to the RCL, it is curved towards the dorsoradial aspect of the joint. Sensory branches of the radial nerve are identified and protected and skin flaps are elevated to demonstrate the extensor hood into which the adductor aponeurosis inserts.

For access to the UCL, an incision is made parallel to the extensor pollicis longus along its ulnar border. The hood mechanism is then retracted to allow direct visualisation of the UCL. A Stener lesion if present, will be seen to lie above the adductor aponeurosis before incision to this layer.

For access to the RCL, the extensor hood is divided between the extensor pollicis longus and brevis tendons. This allows radial reflection of the hood and visualisation of the RCL.

Primary repair of midsubstance tears are repaired end to end using a 3-0 non-absorbable suture. Avulsion injuries require reattachment and bone anchors are now more commonly used than pull-out wires or sutures due to the improved range of motion and pinch strength they offer [28]. The extensor hood is then closed to achieve maximal stability and functional outcome.

The complications that are most frequently encountered in primary repair of the UCL are neuropraxia (6.5\%), stiffness (4\%) and chronic regional pain syndrome (1.6\%) [29]. It would be expected that a similar complication rate would occur with repair of the RCL.

\section{Stener lesions}

The indications for operation have already been outlined and Stener lesions refer to a special type of complete distal UCL tear. In a Stener lesion, as the UCL tears it retracts and comes to lie superficial to the adductor aponeurosis. The interposition maintains separation between the ruptured ends of the ligament thus preventing ligamentous healing. It is this for this reason that Stener lesions should be operatively managed. A RCL Stener-equivalent has been noted when the avulsed distal end of the RCL comes to lie superficial to a well-developed abductor aponeurosis [1]. This however, is very rare and is suspected only following imaging, direct visualisation at surgery or when conservative measures have failed.

\section{Avulsion fractures}

Avulsion fractures represent a special class of collateral ligament injury (see Fig. 5). They cannot be graded I-III since technically there the ligament is not torn. However, it still deserves mention since fractures can compromise the bone-ligament-bone stabilisation complex and can lead to chronic symptoms. The comparative frequency of UCL injuries compared to RCL injuries means that evidence regarding management of avulsion injuries is based upon findings for the ulnar side only. Avulsion fractures involving the UCL have a non-union rate of $25-60 \%$ [30,

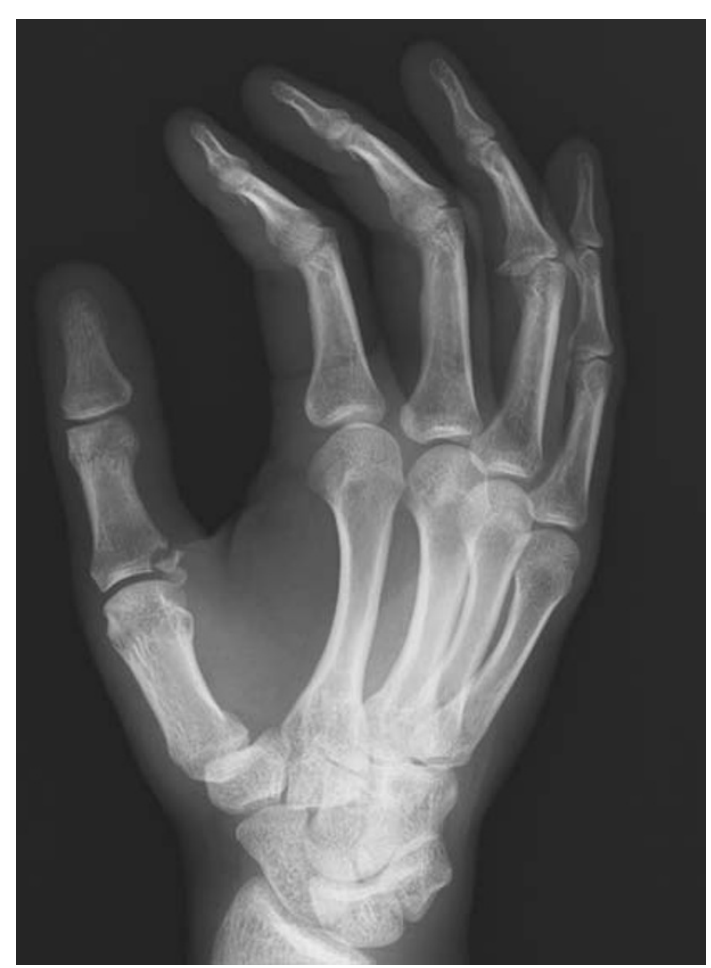

Fig. 5 Radiograph showing a bony avulsion injury 
31 ] and even minimally displaced fractures of $<2 \mathrm{~mm}$ can undergo a significant rotational deformity [32]. Both of these effects can cause painful thumb movements and are arguments for always treating avulsion injuries operatively. However, Sorene and Goodwin [31] investigated displaced fractures that were stable on examination and found that despite $7 \%$ of patients noting pain on thumb movement at least 1 year post-injury, all patients were satisfied. We recommend that only unstable, displaced fractures should be treated operatively whilst undisplaced fractures and stable, displaced fractures should be managed with immobilisation and therapy alone.

\section{Complete tears}

Complete ligamentous tears have been discussed in the literature for over 40 years yet management remains controversial. It has been traditionally regarded that complete UCL injuries should be repaired [25, 33] (due to the high likelihood of a concurrent Stener lesion), while complete RCL injuries should be managed conservatively with immobilisation (since no physical block exists to prevent healing of the two ends of the rupture). There is a however, a recent trend towards operative management of complete RCL injuries whilst one author [26] suggests that complete UCL injuries could be managed non-operatively.

The argument for the non-operative management approach to UCL injuries is thus: Landsman et al. [26] splinted all acute, complete ulnar collateral ligaments for 8-12 weeks and reported $85 \%$ of their cohort of 40 patients healing without significant instability, arthritis, pain, or stiffness (range of motion within $80 \%$ of the contralateral hand). They argued all complete UCL tears should undergo a trial of immobilisation with surgery only needed if symptoms persist.

In contrast, Lane [33] recommended surgery on all complete ruptures making a comparable cohort of 36 patients. 32 of 36 patients accepted early surgery and it noted that $97 \%$ of these patients had a Stener lesion. Of the 4 remaining patients, 2 remained symptomatic and went on to have reconstructive surgery whilst the remaining two were unstable on examination but asymptomatic. It remains unknown whether the unstable asymptomatic patients would go on become symptomatic later. On the basis of Lane's [33] findings, we also recommend operative management for complete UCL injuries.

Treatment of grade III RCL injuries is controversial. Stener lesion equivalents are extremely rare and theoretically without the risk of soft-tissue interposition, the RCL should heal with immobilisation alone [10]. However, surgery is increasingly advocated because complete RCL disruption allows the extensor pollicis longus tendon to distract the MCP joint in an ulnar fashion. The RCL can then heal in a lax position giving rise to late symptomatic instability and degenerative joint disease [34].

Early versus late repair of grade III injuries

Since some surgeons will favour immobilisation for grade III injuries, it is important to know that surgical options for symptomatic patients will change as a result of a delayed procedure. Primary repair of the ligament has traditionally been recommended for injuries less than 3 weeks old while reconstruction advocated for chronic injuries or where there is at least 3 weeks between injury and surgery [25]. It is thought that scar tissue formation and degeneration of ligament ends predisposes to a high rate of failure with a delayed direct repair $[5,25,35,36]$ though this belief is not universal $[37,38]$. The practice of Fairhurst and Hansen [37] was to inspect the remnants of the UCL and directly repair them unless they thought them to be of poor quality, in which case they performed a reconstruction using the extensor pollicis brevis tendon. They reported only one rupture of their group of 13 patients in a patient who had suffered further injury to their hand. Accordingly, we recommend that late direct repair can be performed for UCL and RCL injuries but only if the injury sites are refreshed and healthy tissue is macroscopically present that will allow healing.

\section{Classification of reconstruction}

Reconstruction can be classed in one of two ways:

- Local tissue reconstruction versus free tendon grafting from a distal site.

- Static versus dynamic reconstruction.

Local tissue reconstruction offers advantages in preserving the blood supply of the reconstructed tissue whilst avoiding a separate incision and partial defunctioning of other structures. Free grafts meanwhile give flexibility in graft placement and prevent local tissue trauma at an already injured site [39].

Static reconstructive surgery replaces the injured collateral ligament to prevent deviation and is most commonly comprised of tendon grafts; either local or free. Dynamic reconstruction techniques use a local musculotendinous unit to prevent deviation of the phalanx when the thumb is placed under stress [40].

\section{Dynamic reconstruction}

Dynamic reconstruction of the RCL is more widely described than for the UCL which is attributable to the required outcomes of surgery. MCP joint deviation 
resulting from a RCL injury is less important to pinch and grip function than the UCL injuries. Although dynamic reconstruction is not as stable as static reconstruction, it is technically easier to perform and thus may suffice.

Due to the differing anatomy on each side of the thumb, dynamic reconstruction will be covered separately for RCL and UCL injuries. For UCL injuries, insertion of the extensor indicis proprius tendon into the sagittal fibres of the extensor mechanism ulnar and parallel to the EPL tendon has been described in one patient [36] whilst another limited study reported satisfactory outcomes with detachment of the extensor pollicis brevis tendon from the dorsal base of the proximal phalanx and inserting it into a drill hole on the ulnar aspect of the base of the proximal phalanx [41]. Nevasier et al. [42] and McCue et al. [43] have both reported favourable results in 31 out of 33 patients with combined adductor pollicis tendon detachment and proximal advancement on the proximal phalanx.

For RCL injuries, the abductor pollicis brevis (APB) has been well documented as a dynamic stabiliser. Both Camp et al. [11] and Durham et al. [12] have described advancing the APB tendon though this associated it with pain and loss of motion. More recently, Horch et al. [44] describe a method of leaving the APB insertion in place but pulling the fibres dorsally to change the vector of the tendon to help recreate the stability conferred by the RCL.

\section{Static reconstruction}

The most widely used configurations for static graft reconstruction are a figure of eight configuration (see Fig. 6), a parallel configuration (see Fig. 7), triangular configuration with a proximal apex (see Fig. 8), and a triangular configuration with a distal apex (see Fig. 9). The first three of these configurations use a free tendon; palmaris longus is used most commonly. When absent, a portion of the flexor carpi radialis tendon is taken instead. The last configuration as described by Fairhurst and Hansen [37] to reconstruct the UCL only uses a slip of the

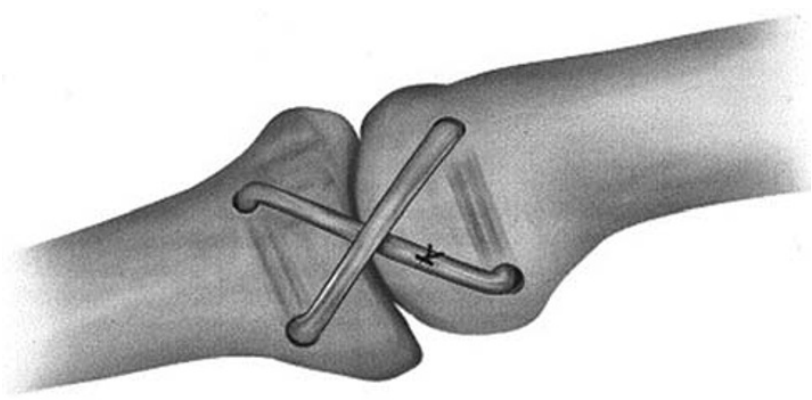

Fig. 6 Reconstruction with a figure of eight configuration (reproduced with permission Hogan et al. 2005)

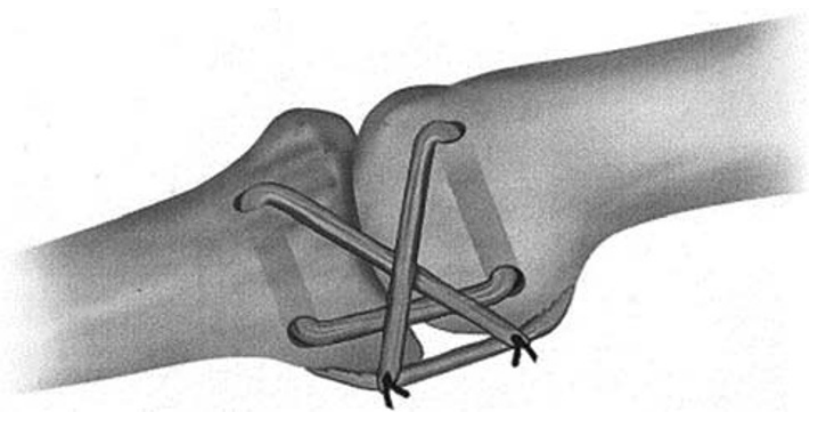

Fig. 7 Reconstruction with a parallel configuration (reproduced with permission Hogan et al. 2005)

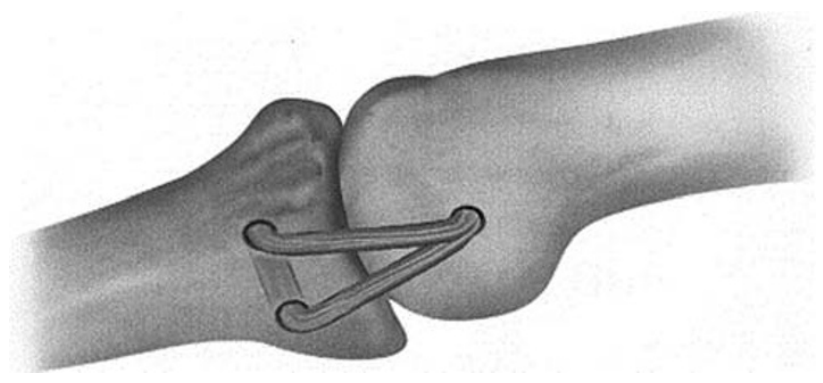

Fig. 8 Reconstruction with a triangular configuration with a proximal apex (reproduced with permission Hogan et al. 2005)

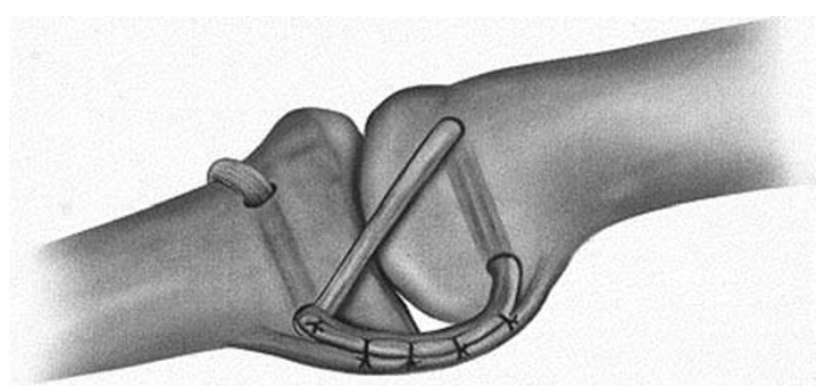

Fig. 9 Reconstruction with a triangular configuration with a distal apex (reproduced with permission Hogan et al. 2005)

extensor pollicis brevis tendon and is an example of a local static reconstruction.

The figure of eight configuration was described by Strandell [45] who passed free graft through bone tunnels and the sutured the graft to itself. Osterman et al. [46] however, noted that this technique caused hyperextension laxity and so modified it to create a parallel configuration by suturing the graft to the volar plate. In 1993, Glickel et al. [47] sought to duplicate the normal anatomy of the UCL; namely a narrow origin with a broad insertion extending dorsal to volar on the ulnar base of the proximal phalanx. Free tendon graft is passed dorsal to volar through a tunnel in the proximal phalanx and both limbs are passed through a single metacarpal tunnel. The free ends of the tendon were originally tied together to make an 
interference knot but a recent modification involves suturing the knot to the periosteum through a radial incision with the option of further securing of the fix with a bone anchor [40]. In 2002, Fairhurst and Hansen [37] described a triangular configuration with a distal apex using a $6 \mathrm{~cm}$ length of the extensor pollicis brevis tendon preserving its distal attachment, and thereby eliminating the need for harvesting a free graft. The tendon was routed through a drill hole from radial to ulnar, across the base of the proximal phalanx and exited at the anatomical site of insertion of the ulnar collateral ligament. It was then passed through a drill hole running from the dorsal to the palmar surface of the metacarpal head and then back to its point of emergence from the base of the proximal phalanx.

The question of which configuration in vivo is best has not been compared and evaluated clinically though cadaveric studies show that Glickel et al.'s [47] triangular configuration with a proximal apex offers the greatest range of motion and stability. Lee et al. [48] demonstrated that this was the only configuration to restore range of motion in the plane of flexion and extension comparably to the uninjured side. Furthermore, Hogan et al. [49] demonstrated that whilst no reconstruction could fully replicate the strength of an uninjured UCL, the triangular configuration with a proximal apex using an interference knot fails at a much higher moment than the other configurations thereby providing the strongest reconstruction.

With respect specifically to RCL reconstruction, the triangular configuration with a proximal apex has been shown to have comparable outcomes on joint motion and stability at the MCP joint, grip and pinch strength as early repair of the ligament [13].

Wong et al. [50] have recently suggested using a boneperiosteum-bone (BPB) graft as an alternative to the traditional use of tendon. In seven patients with chronic UCL instability, they excised the capsular and ligamentous remnants and burred the bone to a depth of $8 \mathrm{~mm}$. A BPB graft was harvested from the iliac crest and the bone blocks were fixed to the burred sites with the periosteum crossing the joint to replicate the UCL. The results are definitely encouraging though use in a larger cohort is needed before being universally advocated. Pending this, we recommend static reconstruction with a triangular configuration with a proximal apex as the most appropriate means of reconstruction of the UCL and RCL due to the restoration in function it is likely to confer.

\section{Degenerative joint disease}

Evidence of MCP joint degeneration noted radiographically or intra-operatively will likely limit any anticipated improvement by repairing or reconstructing a MCP collateral ligament. In these instances, fusion of the MTP joint has been advocated [38, 42, 51] though it is the authors' opinion that securely fastened joint arthroplasty offers a viable alternative.

\section{Treatment algorithm}

On the basis of the current literature, it is our opinion that RCL and UCL injuries can be managed in a similar manner according to the treatment algorithm outlined in Fig. 10. We believe static reconstruction to offer advantages over dynamic options, and the use of a triangular configuration with proximal apex is advocated.

\section{Conclusions}

Instability at the MCP joint arises can arise after an acute, forced injury or following repeated trauma. History, examination and plain radiographs form the basis of a

Fig. 10 Treatment algorithm

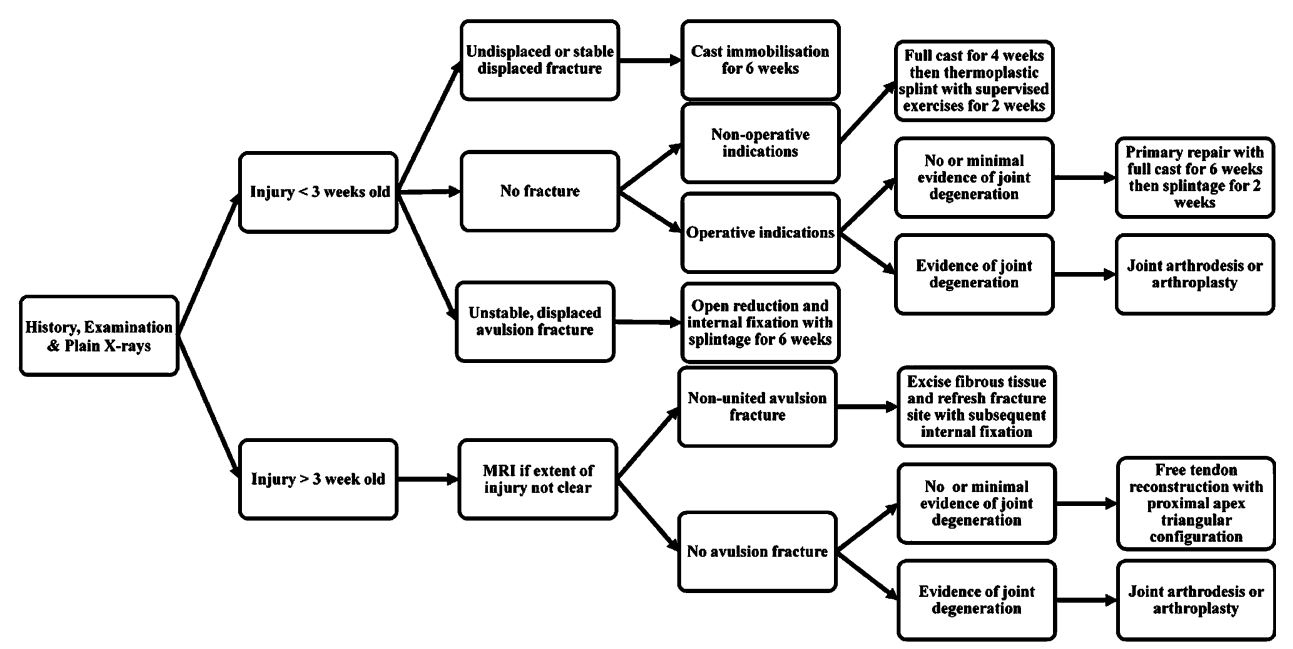


treatment algorithm that can be used for both UCL and RCL injuries. Accurate and effective management is important to prevent long-term morbidity and restore function.

\section{References}

1. Coyle MP Jr (2003) Grade III radial collateral ligament injuries of the thumb metacarpophalangeal joint: treatment by soft tissue advancement and bony reattachment. J Hand Surg [Am] 28:1420

2. Joseph J (1951) Further studies of the metacarpo-phalangeal and interphalangeal joints of the thumb. J Anat 85:221-229

3. Ebrahim FS, De Maeseneer M, Jager T, Marcelis S, Jamadar DA, Jacobson JA (2006) US diagnosis of UCL tears of the thumb and Stener lesions: technique, pattern-based approach, and differential diagnosis. Radiographics 26:1007-1020

4. Lyons RP, Kosin SH, Failla JM (1998) The anatomy of the radial side of the thumb: static restraints in preventing subluxation and rotation after injury. Am J Orthop 27:759-763

5. Frank WE, Dobyns J (1972) Surgical pathology of collateral ligamentous injuries of the thumb. Clin Orthop Relat Res 83:102114

6. Krause JO, Manske PR, Mirly HL, Szerzinski J (1996) Isolated injuries to the dorsoradial capsule of the thumb metacarpophalangeal joint. J Hand Surg [Am] 21:428-433

7. Bean CH, Tencer AF, Trumble TE (1999) The effect of thumb metacarpophalangeal ulnar collateral ligament attachment site on joint range of motion: an in vitro study. J Hand Surg [Am] 24:283-287

8. Stener B (1962) Displacement of the ruptured ulnar collateral ligament of the metacarpo-phalangeal joint of the thumb. J Bone Joint Surg [Br] 44-B:869-879

9. Gerber C, Senn E, Matter P (1981) Skier's thumb. Surgical treatment of recent injuries to the ulnar collateral ligament of the thumb's metacarpophalangeal joint. Am J Sports Med 9:171-177

10. Campbell CS (1955) Gamekeeper's thumb. J Bone Joint Surg [Br] 37-B:148-149

11. Camp RA, Weatherwax RJ, Miller EB (1980) Chronic posttraumatic radial instability of the thumb metacarpophalangeal joint. J Hand Surg [Am] 5:221-225

12. Durham JW, Khuri S, Kim MH (1993) Acute and late radial collateral ligament injuries of the thumb metacarpophalangeal joint. J Hand Surg [Am] 18:232-237

13. Catalano LW III, Cardon L, Patenaude N, Barron OA, Glickel SZ (2006) Results of surgical treatment of acute and chronic grade III tears of the radial collateral ligament of the thumb metacarpophalangeal joint. J Hand Surg [Am] 31:68-75

14. Malik AK, Morris T, Chou D, Sorene E, Taylor E (2009) Clinical testing of ulnar collateral ligament injuries of the thumb. J Hand Surg [Eur] 34:363-366

15. Melone CP Jr, Beldner S, Basuk RS (2000) Thumb collateral ligament injuries. An anatomic basis for treatment. Hand Clin 16:345-357

16. Heyman P, Gelberman RH, Duncan K, Hipp JA (1993) Injuries of the ulnar collateral ligament of the thumb metacarpophalangeal joint. Biomechanical and prospective clinical studies on the usefulness of valgus stress testing. Clin Orthop Relat Res 292:165-171

17. Abrahamsson SO, Sollerman C, Lundborg G, Larsson J, Egund N (1990) Diagnosis of displaced ulnar collateral ligament of the metacarpophalangeal joint of the thumb. J Hand Surg [Am] $15: 457-460$
18. Cooper JG, Johnstone AJ, Hider P, Ardagh MW (2005) Local anaesthetic infiltration increases the accuracy of assessment of ulnar collateral ligament injuries. Emerg Med Australas 17:132136

19. Bronstein AJ, Koniuch MP, von Holsbeeck M (1994) Ultrasonographic detection of thumb ulnar collateral ligament injuries: a cadaveric study. J Hand Surg [Am] 19:304-312

20. Louis DS, Buckwalter KA (1989) Magnetic resonance imaging of the collateral ligaments of the thumb. J Hand Surg [Am] 14:739741

21. Palmer AK, Louis DS (1977) Gamekeeper's thumb. When is surgery necessary? Orthop Trans 1:14-15

22. Louis DS, Huebner JJ Jr, Hankin FM (1986) Rupture and displacement of the ulnar collateral ligament of the metacarpophalangeal joint of the thumb. Preoperative diagnosis. J Bone Joint Surg $[\mathrm{Am}]$ 68-A:1320-1326

23. Hintermann B, Holzach PJ, Schütz M, Matter P (1993) Skier's thumb-the significance of bony injuries. Am J Sports Med 21:800-804

24. Pechlaner S (1982) Injuries of the ulnar ligaments of the thumb joint. Aktuelle Traumatol 12:210-214

25. Smith RJ (1977) Post-traumatic instability of the metacarpophalangeal joint of the thumb. J Bone Joint Surg [Am] 59-A:14-21

26. Landsman JC, Seitz WH Jr, Froimson AI, Leb RB, Bachner EJ (1995) Splint immobilization of gamekeeper's thumb. Orthopedics 18:1161-1165

27. Sollerman C, Abrahamsson SO, Lundborg G, Adalbert K (1991) Functional splinting versus plaster cast for ruptures of the ulnar collateral ligament of the thumb. A prospective randomized study of 63 cases. Acta Orthop Scand 62:524-526

28. Kuz JE, Husband JB, Tokar N, McPherson SA (1999) Outcome of avulsion fractures of the ulnar base of the proximal phalanx of the thumb treated nonsurgically. J Hand Surg [Am] 24:275282

29. Chuter GS, Muwanga CL, Irwin LR (2009) Ulnar collateral ligament injuries of the thumb: 10 years of surgical experience. Injury 40:652-656

30. Katolik LI, Friedrich J, Trumble TE (2008) Repair of acute ulnar collateral ligament injuries of the thumb metacarpophalangeal joint: a retrospective comparison of pull-out sutures and bone anchor techniques. Plast Reconstr Surg 122:1451-1456

31. Sorene ED, Goodwin DR (2003) Non-operative treatment of displaced avulsion fractures of the ulnar base of the proximal phalanx of the thumb. Scand J Plast Reconstr Surg Hand Surg 37:225-227

32. Dinowitz M, Trumble T, Hanel D, Vedder NB, Gilbert M (1997) Failure of cast immobilization for thumb ulnar collateral ligament avulsion fractures. J Hand Surg [Am] 22:1057-1063

33. Lane LB (1991) Acute Grade III ulnar collateral ligament ruptures. A new surgical and rehabilitation protocol. Am J Sports Med 19:234-237

34. Posner MA, Retaillaud JL (1992) Metacarpophalangeal joint injuries of the thumb. Hand Clin 8:713-732

35. Frykman G, Johansson O (1956) Surgical repair of rupture of the ulnar collateral ligament of the thumb of the metacarpophalangeal joint of the thumb. Acta Chir Scand 112:58-64

36. Kaplan EB (1961) The pathology and treatment of radial subluxation of the thumb with ulnar displacement of the head of the first metacarpal. J Bone Joint Surg [Am] 43-A:541-546

37. Fairhurst M, Hansen L (2002) Treatment of "Gamekeeper's Thumb" by reconstruction of the ulnar collateral ligament. J Hand Surg [Br] 27:542-545

38. Coonrad RW, Goldner JL (1968) A study of the pathological findings and treatment in soft-tissue injury of the thumb metacarpophalangeal joint. With a clinical study of the normal range of motion in one thousand thumbs and a study of post mortem 
findings of ligamentous structures in relation to function. $\mathrm{J}$ Bone Joint Surg [Am] 50-A:439-451

39. Edelstein DM, Kardashian G, Lee SK (2008) Radial collateral ligament injuries of the thumb. J Hand Surg [Am] 33:760-770

40. Glickel SZ (2002) Thumb metacarpophalangeal joint ulnar collateral ligament reconstruction using a tendon graft. Tech Hand Up Extrem Surg 6:133-139

41. Sakellarides HT (1978) Treatment of recent and old injuries of the ulnar collateral ligament of the MP joint of the thumb. Am J Sports Med 6:255-262

42. Neviaser RJ, Wilson JN, Lievano A (1971) Rupture of the ulnar collateral ligament of the thumb (gamekeeper's thumb). Correction by dynamic repair. J Bone Joint Surg [Am] 53-A:1357-1364

43. McCue FC 3rd, Hakala MW, Andrews JR, Gieck JH (1974) Ulnar collateral ligament injuries of the thumb in athletes. J Sports Med 2:70-80

44. Horch RE, Dragu A, Polykandriotis E, Kneser U (2006) Radial collateral ligament repair of the thumb metacarpophalangeal joint using the abductor pollicis brevis tendon. Plast Reconstr Surg 117:491-496

45. Strandell G (1959) Total rupture of the ulnar collateral ligament of the metacarpophalangeal joint of the thumb. Acta Chir Scand 118:72-80

46. Osterman AL, Hayken GD, Bora FW Jr (1981) A quantitative evaluation of thumb function after ulnar collateral repair and reconstruction. J Trauma 21:854-861

47. Glickel SZ, Malerich M, Pearce SM, Littler JW (1993) Ligament replacement for chronic instability of the ulnar collateral ligament of the metacarpophalangeal joint of the thumb. J Hand Surg [Am] 18:930-941

48. Lee SK, Kubiak EN, Lawler E, Iesaka K, Liporace FA, Green SM (2005) Thumb metacarpophalangeal ulnar collateral ligament injuries: a biomechanical simulation study of four static reconstructions. J Hand Surg [Am] 30:1056-1060

49. Hogan CJ, Ruland RT, Levin LS (2005) Reconstruction of the ulnar collateral ligament of the thumb metacarpophalangeal joint: a cadaver study. J Hand Surg [Am] 30:394-399

50. Wong TC, Ip FK, Wu WC (2009) Bone-periosteum-bone graft reconstruction for chronic ulnar instability of the metacarpophalangeal joint of the thumb-minimum 5-year follow-up evaluation. J Hand Surg [Am] 34:304-308

51. Moberg E, Stener B (1953) Injuries to the ligaments of the thumb and fingers; diagnosis, treatment and prognosis. Acta Chir Scand 106:166-186

52. Bowers WH, Hurst LC (1977) Gamekeeper's Thumb. J Bone Joint Surg [Am] 59-A:519-524

53. Palmer AK, Louis DS (1978) Assessing ulnar instability of the metacarpophalangeal joint of the thumb. J Hand Surg [Am] 3:542-546

54. Derkash RS, Matyas JR, Weaver JK, Oden RR, Kirk RE, Freeman JR, Cipriano FJ (1987) Acute surgical repair of the skier's thumb. Clin Orthop Relat Res 216:29-33

55. Musharafieh RS, Bassim YR, Atiyeh BS (1997) Ulnar collateral ligament rupture of the first metacarpophalangeal joint: a frequently missed injury in the emergency department. J Emerg Med 15:193-196 